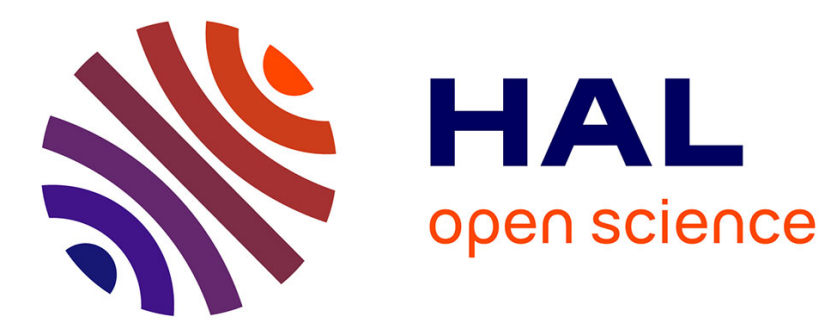

\title{
Faceted Fresnel DOEs creating the perception of a floating 3D virtual object under divergent illumination
} Qiang Song, Yoran Eli Pigeon, Kevin Heggarty

\section{To cite this version:}

Qiang Song, Yoran Eli Pigeon, Kevin Heggarty. Faceted Fresnel DOEs creating the perception of a floating 3D virtual object under divergent illumination. Optics Communications, 2019, 451, pp.231239. 10.1016/j.optcom.2019.06.047 . hal-02189529

\section{HAL Id: hal-02189529 \\ https://imt-atlantique.hal.science/hal-02189529}

Submitted on 19 Jul 2019

HAL is a multi-disciplinary open access archive for the deposit and dissemination of scientific research documents, whether they are published or not. The documents may come from teaching and research institutions in France or abroad, or from public or private research centers.
L'archive ouverte pluridisciplinaire HAL, est destinée au dépôt et à la diffusion de documents scientifiques de niveau recherche, publiés ou non, émanant des établissements d'enseignement et de recherche français ou étrangers, des laboratoires publics ou privés. 


\title{
Faceted Fresnel DOEs creating the perception of a floating 3D virtual object under divergent illumination
}

\author{
Qiang Song, Yoran Eli Pigeon, Kevin Heggarty \\ Department of Optic, IMT-Atlantique, Technopole Brest-Iroise, CS 83818,29285 BREST, France \\ E-mail: qiang.song@imt-atlantique.fr
}

Abstract: An approach for the optimization and fabrication of a phase-only faceted Fresnel type diffractive optical element (FDOE) creating 3D virtual object is proposed. The FDOE is a transmissive Fresnel type DOE array, which produces the perception of a customized floating 3D virtual object behind the FDOE when illuminated with a divergent monochromatic Light Emitter Diode (LED) source. Each DOE unit of the FDOE is optimized by a modified iterative Fourier transform algorithm (M-IFTA). Every unit of the FDOE locally deflects the incident light to the same position to form a designated view in the target plane. The FDOE is fabricated using our home-built parallel writing photo-lithography machine. Numerical simulations and optical experiments are performed to verify the proposed design method. This work may find important applications in the advanced design of optical security hologram and anti-counterfeiting component.

Keywords: Micro optics; Diffractive optics; Iterative algorithm; Binary Optics; Photo-Lithography.

\section{Introduction}

Security holograms are one of the most important optical security technologies, which are widely used in anticounterfeiting protection of bank notes, passport and ID cards [1-12]. There are two main approaches for producing security holograms: one is based on the original holography technique, using light-sensitive materials to record the optical interference fringes with an optical interferometer system. This requirement makes it difficult to produce nonphysical or virtual objects, and to address cost-effective mass production. The other main approach uses surface relief diffractive optical elements (DOEs), whose advantage compared to optically recorded holograms is that they do not require the interferometer system. This enables mathematical models of diffraction processes to be used with computer programming, which is more suitable for making optical security holograms. Security holograms which can produce new visual perception are still desperately needed for modern anti-counterfeiting protection. What fundamentally new visual perception effect can we expect?

A DOE generating perceived floating 3D virtual object under divergent illumination might be a good candidate, because 3D floating virtual object can provide observers a perception close to real life. In addition, a LED source is universally available in daily life, and safety for the human eye. Several related research literatures on this aspect have appeared in recent years. For instance, Anton Goncharsky and his co-authors proposed a subwavelength grating array method using electron-beam lithography (EBL) technology that can generate visual 2D and 3D images on the surface of the DOE plane [13-15]. This can give an observer an impressive 3D vision effect, but, not a floating visual effect. In addition, the diffraction efficiency of the gratings is generally low, and the higher orders can produce crosstalk. Multilevel gratings or blazed gratings are an effective way to improve the diffraction efficiency, but the fabrication process is very complicated with EBL. Later, the same team used a similar method to realize 360 degree 3D image with grating arrays fabricated on a cylindrical surface [16]. Furthermore, a recent study presented a frequency mosaic method using maskless lithography which can produce 3D visual perception [17]. Nevertherless, 
the diffraction efficiency is low and faraway LED illumination is needed to reproduce the virtual object on the surface of the hologram.

In this work we propose a new way to produce a floating 3D virtual image with divergent LED source, which we name faceted pure phase Fresnel DOEs. To the best of our knowledge, this is the first time it is being reported to the optics community. LED sources are used because they are less dangerous for visual perception than lasers, and are also more convenient, as no beam-expanding optics are required, since it is a divergent light source. On the surface of the FDOE, each DOE facet diffracts incident light to generate different virtual viewing angle images toward to the observer position; it therefore belongs to the off-axis type DOE. To obtain high image quality and diffraction efficiency, a modified iterative Fourier transform algorithm (IFTA) is used to optimize each DOE facet. After design, the FDOE is fabricated with our home-built gray scale direct parallel writing photolithography machine. Gray-scale maskless photolithography is an effective technique for fabricating high quality complicated micro relief structures, which can help increase DOE's diffraction efficiency. When the FDOE is illuminated by a divergent LED source, a floating 3D virtual image is formed behind the FDOE; an observer can perceive different views of the virtual 3D object hanging in space. The configuration is described in detail in Section 2. Section 3 is devoted to illustrating the design procedure. Fabrication and experiment results are presented in Section 4, and results are discussed in Section 5. Finally, we conclude our work in Section 6.

\section{Principle and configuration}

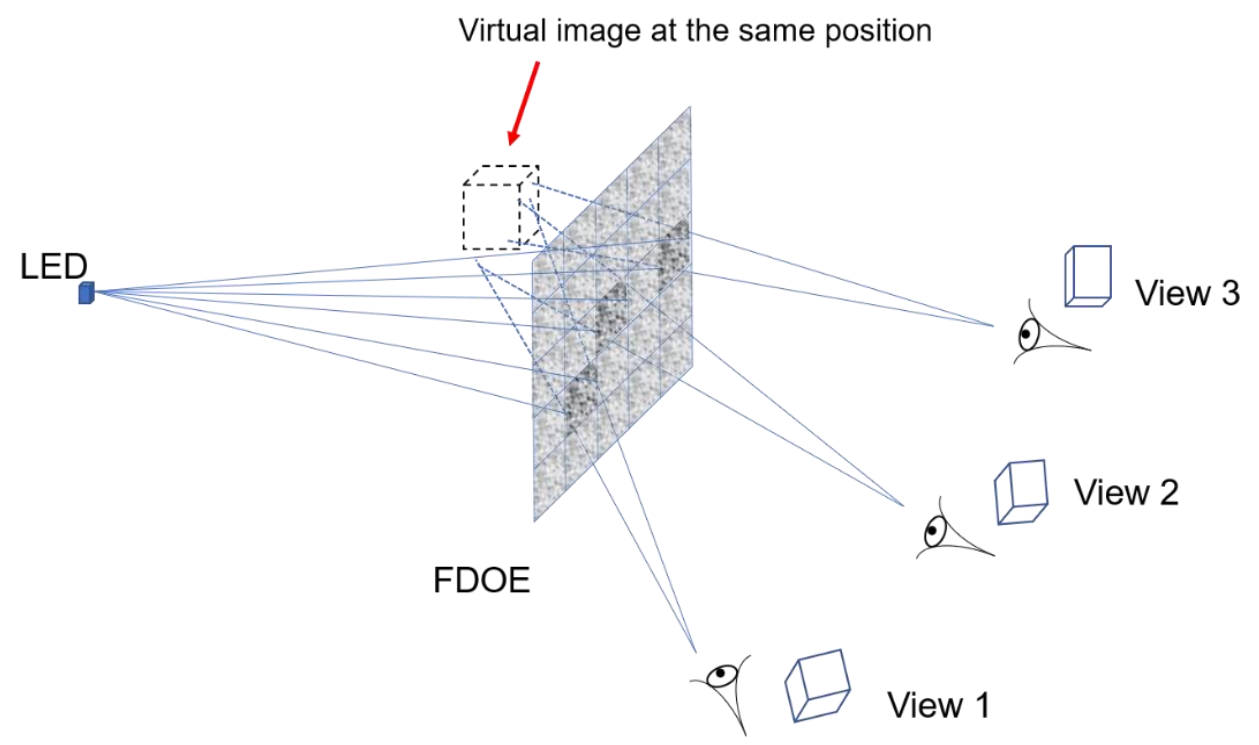

Fig.1. The configuration of the LED source and observer

The essential idea of the method proposed in this work is sketched in Fig.1. Fig.1 describes the layout of observations which comprises the LED source and FDOE. The LED source is behind the FDOE. To produce the 3D desired visual effect, we propose an approach based on faceted off-axis Fresnel DOEs. In such an approach the overall diffractive structure is separated into a matrix of individual Fresnel DOEs. Each facet diffracts to generate a view of the same basic 3D object, but seen from a slightly different angle. By carefully optimizing the angles for each facet, when an observer moves their eye from side to side at the FDOE plane, they observe the different views, generating the slightly different angular views of the 3D object. The overall effect is that of observing a real 3D object. To make sure the observer has the impression that each view comes from the same object in the same position, the different DOE facets must generate their reconstructed images with the correct off-axis offset. The lateral displacement of each reconstructed image should be calculated carefully. The basic calculation principle is shown below in Fig.2. 
The global coordinates of the FDOE plane are denoted by $\left(x_{0}, y_{0}\right)$, and the local coordinates of the m-th DOE are denoted by $\left(x_{m}, y_{m}\right)$. The distance between the FDOE plane and the virtual image plane is denoted by $-\mathrm{z}$, where $\mathrm{z}$ is a positive value, the distance is negative. The complex amplitude distribution of $(m, n)$-th DOE on FDOE plane can be described as followings:

$$
H_{m, n}\left(x_{m}, y_{n}\right)=A_{\text {source }} \exp \left[j \varphi_{m, n}\right]
$$

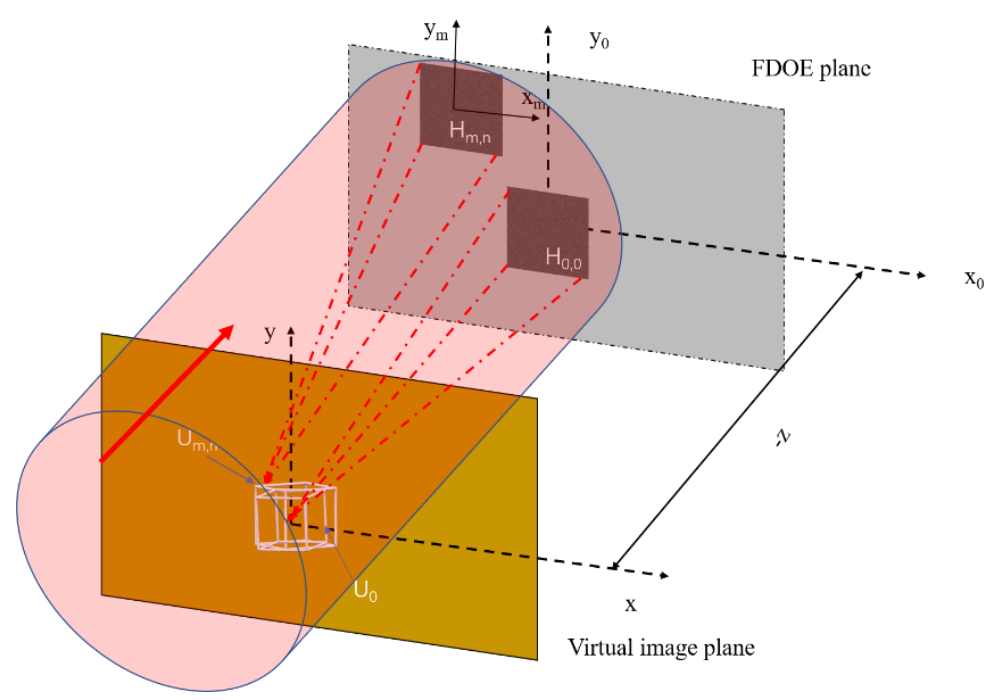

Fig.2. Principle of proposed FDOE calculation process

where $A_{\text {source }}$ and $\varphi_{m}$ are the amplitude of the incident light and, the m-th DOE's phase distribution, respectively. According to Fresnel diffraction theory, the diffracted field distribution of the $(0,0)$-th DOE in the virtual image plane can be calculated by

$$
U_{0}(\mathrm{x}, \mathrm{y})=\frac{\exp (-j k z)}{-j \lambda z} \exp \left[j \frac{k}{-2 z}\left(x^{2}+y^{2}\right)\right] \iint H_{0,0} e^{\frac{j k}{-2 z}\left[x_{0}{ }^{2}+y_{0}{ }^{2}\right]} d x_{0} d y_{0}
$$

In eq.(2), $\mathrm{k}=2 \pi / \lambda$ is the wave number in free space, $\lambda$ is the design wavelength, and $x_{0}, y_{0}, x, y$, are the coordinates axis in Fig.2, respectively. In order to generate the reconstructed virtual image of the (m, n)-th DOE toward to the same position as that of the (0,0)-th DOE, eq.(2) should be modified. See in Fig.2, $\mathrm{x}_{\mathrm{m}}$ and $\mathrm{y}_{\mathrm{n}}$ can be expressed as

$$
\begin{gathered}
x_{m}=x_{0}+m P_{D O E} \\
y_{n}=y_{0}+n P_{D O E}
\end{gathered}
$$

In eq.(3) and eq.(4), $P_{D O E}$ represents the facet size of the FDOE, $m$ and $n$ are the displacement along the $\mathrm{x}_{0}$ axis and the $\mathrm{y}_{0}$ axis, respectively. According to Fresnel diffraction theory, the complex amplitude distribution $U_{\mathrm{m}, \mathrm{n}}(\mathrm{x}, \mathrm{y})$ in the virtual image plane contributed by the wavefront from the $(m, n)$-th DOE can be calculated as

$$
U_{m, n}(\mathrm{x}, \mathrm{y})=\frac{\exp (-j k z)}{-j \lambda z} \iint H_{m, n}\left(x_{m}, y_{n}\right) e^{\frac{j k}{-2 z}\left[\left(x-x_{m}\right)^{2}+\left(y-y_{n}\right)^{2}\right.} d x_{m} d y_{n}
$$

Substituting eq.(3) and eq.(4) into eq.(5),

$$
\begin{gathered}
U_{m, n}(\mathrm{x}, \mathrm{y})=\frac{e^{-j k z}}{-j \lambda z} \mathrm{e}^{\left[\frac{\mathrm{jk}}{-2 \mathrm{z}}\left(\mathrm{x}^{2}+\mathrm{y}^{2}\right)\right]} e^{\frac{j k\left(m P_{D O E} x+n P_{D O E} y\right)}{z}} \\
\times \iint H_{m, n}\left(x_{m}, y_{n}\right) e^{\frac{j k}{-2 \mathrm{z}}\left[\left(x_{0}+m P_{D O E}\right)^{2}+\left(y_{0}+n P_{D O E}\right)^{2}\right]} e^{\frac{j k}{-2 \mathrm{z}}\left(x x_{0}+y y_{0}\right)} d x_{0} d y_{0}
\end{gathered}
$$

Where, $f_{x}, f_{y}$ are defined as

$$
f_{x}=\frac{x}{\lambda z} \quad f_{y}=\frac{y}{\lambda z}
$$


With the 2D Fourier transform, eq.(6) can be simplified as

$$
U_{m, n}(\mathrm{x}, \mathrm{y})=\frac{e^{-j k z}}{-j \lambda z} e^{\frac{j \mathrm{k}}{-2 \mathrm{z}}\left(\mathrm{x}^{2}+\mathrm{y}^{2}\right)} e^{\frac{j k\left(m P_{D O E} x+n P_{D O E} y\right)}{z}} \cdot \mathcal{F}_{2 D}\left[H_{m, n} e^{\frac{j k}{-2 \mathrm{z}}\left[\left(x_{0}+m P_{D O E}\right)^{2}+\left(y_{0}+n P_{D O E}\right)^{2}\right]}\right]
$$

According to eq.(8), the diffracted field of the off-axis Fresnel DOE can still be expressed in a 2D Fourier transform format. Therefore, it can be computed quickly with the fast Fourier transform method. Sampling constraints for the Fresnel diffraction calculation should also be considered. According to the Nyquist sampling rule, the absolute value of the propagation distance $\mathrm{z}$ should satisfy the condition as below[18]:

$$
\mathrm{z}>\frac{N \Delta p^{2}}{\lambda}
$$

Here, in eq.(9), $\mathrm{N}$ is the number of sampling points and $\Delta p$ is the DOE pitch. Generally, it is a phase retrieval problem to get the DOE phase. In this work, a modified iterative Fourier transform algorithm is implemented to design each off-axis Fresnel type DOE, it belongs to the IFTA family [19-22]. The proposed method optimizes the image quality and the fabrication performance simultaneously incorporate with a soft quantization iterative algorithm. The fabricated phase levels are quantized, so the surface relief of the DOE should be divided into several levels. In theory, the higher the number of phase levels, the better the reconstructed image quality and diffraction efficiency. But, the efficiency increase for greater numbers of phase level is often less than the decrease from fabrication errors. Thus, eight-levels is a good practical compromise. The details of the design procedures are provided in Section 3.

\section{Design procedure and numerical simulations \\ 3.1 Design method}

The flowchart of the proposed design approach is shown in Fig.3. The stack of different viewing angle target images is obtained from a 3D model. Each target image is input to the DOE optimization algorithm. This iterative algorithm can be divided into three stages, the so called three-part IFTA. The first stage is the standard Gerchberg-Saxton (GS) algorithm, it is implemented to get the rough phase solution, the GS operation process can be considered as the transformation of signals between the input DOE plane and diffracted image plane.

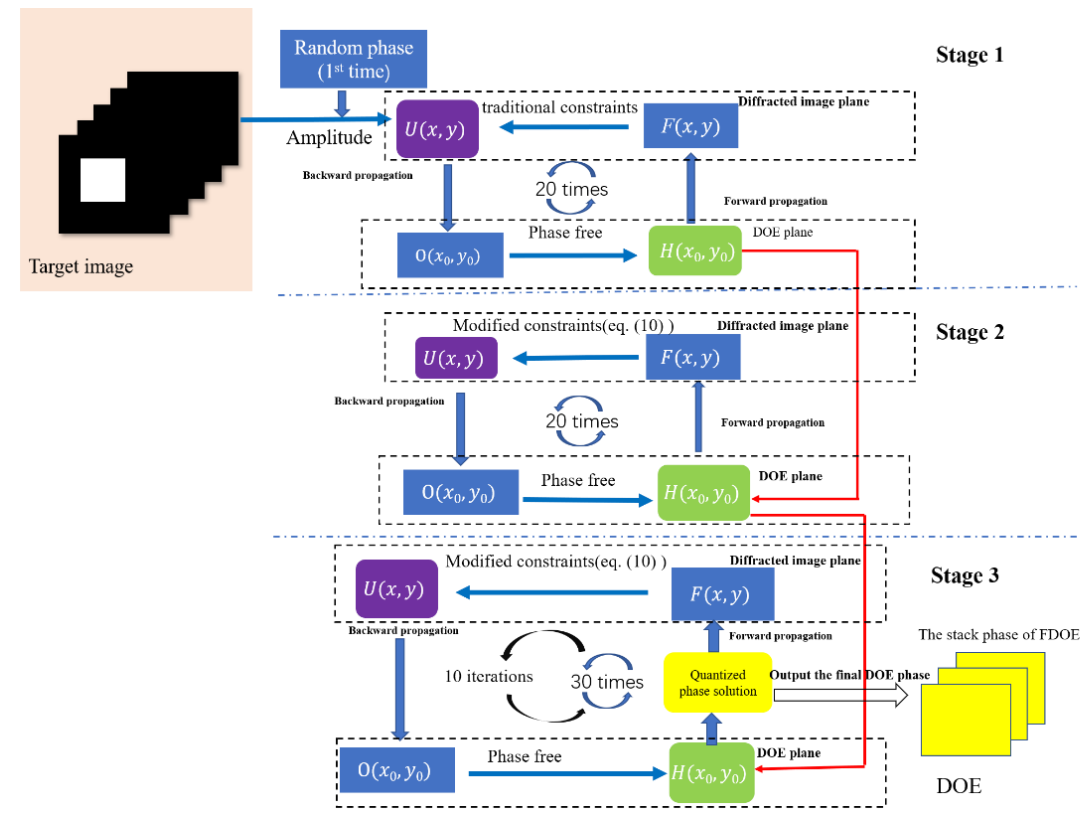

Fig.3. Flowchart of the proposed iterative optimization process of FDOE

The GS iteration process can be described as four basic steps: (1) the process starts from the diffracted image plane. An initial random phase is generated in the range $0-2 \pi$, and then multiplied by an amplitude matrix with the current 
viewing angle target image to form the initial complex amplitude. (2) This field is propagated to the FDOE plane using inverse Fresnel diffraction field propagation. (3) Note that in what follows, we assume that the DOE facets are illuminated by plane waves. Illuminating such a DOE with spherical wave will simply shift the position of the observed image along the optical axis; see discussion part for more details. The amplitude of the wave-front $O\left(x_{0}, y_{0}\right)$ is replaced by the intensity distribution of the source amplitude, while the wavefront phase is retained, to form the updated complex amplitude $H\left(x_{0}, y_{0}\right)$ at the DOE plane. (4) Propagating the result back to the diffracted image plane, the phase obtained from the Fresnel diffraction propagation is combined with the corresponding view image amplitude. The process then repeats from step 1. Based on the above steps, the phase is optimized for 20 iterations, then the algorithm goes on to the second stage, where the iteration process is similar to the GS, but the constraint factor strategy on the diffracted image plane is different to the conventional GS algorithm, which is given as

$$
\left|U_{k}\right|=\left\{\begin{array}{cc}
2 \beta_{k}\left|F_{\text {target }}\right|-\left|F_{k}\right|, & F_{k} \in S \\
\left|F_{k}\right|, & \text { else }
\end{array}\right.
$$

Where, $F_{K}$ is the reconstruction result of the $k$-th iteration, $F_{\text {target }}$ is the target amplitude, $\beta_{k}$ is a weighting factor, and $S$ is the signal area, see Fig.4.

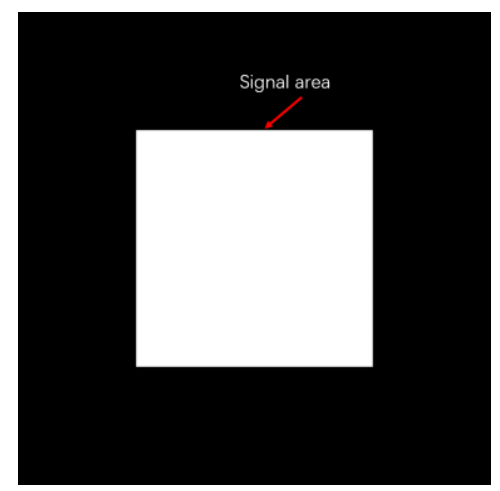

Fig.4. The definition of signal area in the diffracted image plane.

For a more detailed depiction of the modified amplitude constraint, we refer the interested readers to our previous work [20]. After that, the optimization process goes into the third stage; this stage is called the iterative soft quantization method. The soft quantization is a stepwise iterative quantization method. In this part, the iterative process is similar to that of the second stage, but another loop is introduced into it. This process of iteration can be divided into 10 cycles, each cycle includes 30 iterations. The soft quantization operation can be demonstrated as [21] :

First we define $\varphi_{1}$ as

$$
\begin{gathered}
\varphi_{1}=\frac{\varphi_{\text {phase }}^{k}}{\frac{2 \pi}{\text { Levels }}} \\
\varphi_{\text {phase }}^{\prime k}=\varphi_{1}, \text { if }-\frac{\varepsilon_{P}}{2} \leq \varphi_{1}-\operatorname{round}\left(\varphi_{1}\right)<\frac{\varepsilon_{P}}{2}(1<p<10)
\end{gathered}
$$

In eq.(10), $\varphi_{\text {phase }}^{k}$ is the un-quantized phase of the $k$-th iteration, Levels is number of phase levels, $\varphi_{\text {phase }}^{\prime k}$ represents the quantized phase in eq.(12). The quantity $\varepsilon_{P}$ increases with the index $p, p$ is the current loop, the maximum loop is 10 in this work. $p$ is an empirical parameter. Here, the collection of the values in this work is

$$
\begin{gathered}
\varepsilon_{1}=0.15, \varepsilon_{2}=0.3, \varepsilon_{3}=0.45, \varepsilon_{4}=0.6, \varepsilon_{5}=0.7, \varepsilon_{6}=0.8, \varepsilon_{7}=0.85, \\
\varepsilon_{8}=0.9, \varepsilon_{9}=0.95, \varepsilon_{10}=1,
\end{gathered}
$$

The reason why we choose 30 iterations in this process are discussed in next sub-section where the convergence behavior is described. So, in this way, the phase can be quantized into eight levels with high a quality reconstructed image, and adapted to the fabrication constraints. The iterative calculation stops when the error between the intensity 
distribution on the reconstructed plane and target image is small enough, or the maximum number of iterations is reached. The root mean square error (RMSE) can be defined as

$$
\operatorname{RMSE}=\frac{\iint\left(U_{\text {target }}-\gamma\left|U_{K}\right|\right)^{2}}{\iint U_{\text {target }}^{2}}
$$

where $\gamma$ is a scale factor, which can be written as

$$
\gamma=\left(\frac{\iint U_{\text {target }}{ }^{2}}{\iint\left|U_{K}\right|^{2}}\right)
$$

When calculations of all the DOE facets are finished, the set of DOE phases are assembled to get the final FDOE.

\subsection{Numerical simulation}

For the numerical simulations, a 3D "cube" object was used to generate the different viewing angle images. In this study, we use horizontal views. To examine the convergence behavior of the proposed optimization algorithm, one view of these image is selected as the target image, which is shown in Fig.5(a), it is the center view of the 3D object. The RMSE value is plotted against the iteration numbers in Fig.5(b). In this numerical simulation work, the wavelength is $525 \mathrm{~nm}$, the DOE pixel pitch is $0.75 \mu \mathrm{m}$, the number of sampling points is 3600 in each DOE facet, and the distance between the FDOE plane and the virtual image is set as $-120 \mathrm{~mm}$. Acoording to eq.(14), the RMSE curves are calculated with different numbers of iterations in the soft quantization stage. For example, 50 iterations are needed in the soft quantization step, the total number of iteration is 540 (calculated as 20 (the first stage)

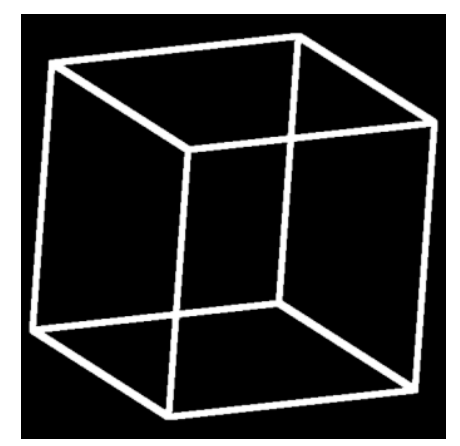

(a) Target image for testing the algorithm's convergence behavior

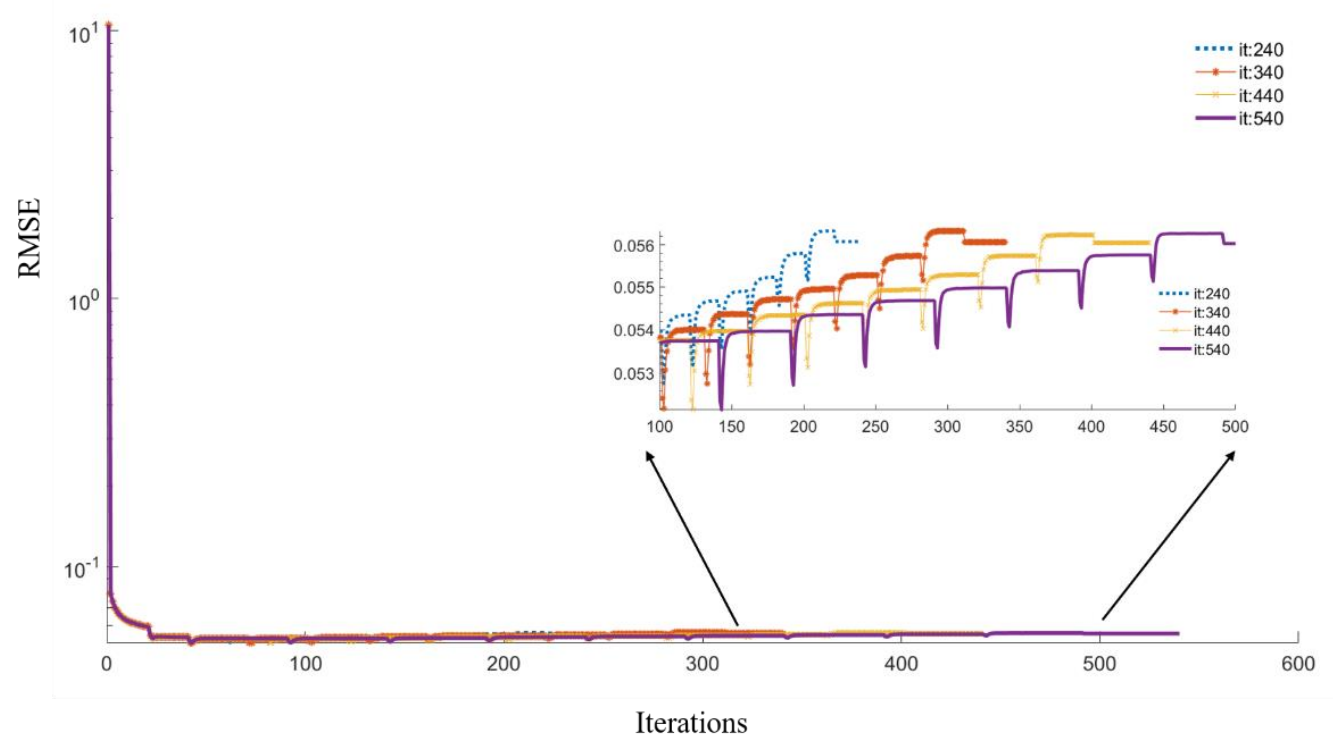

(b) Curve of the reconstruction's RMSE against the iterations 


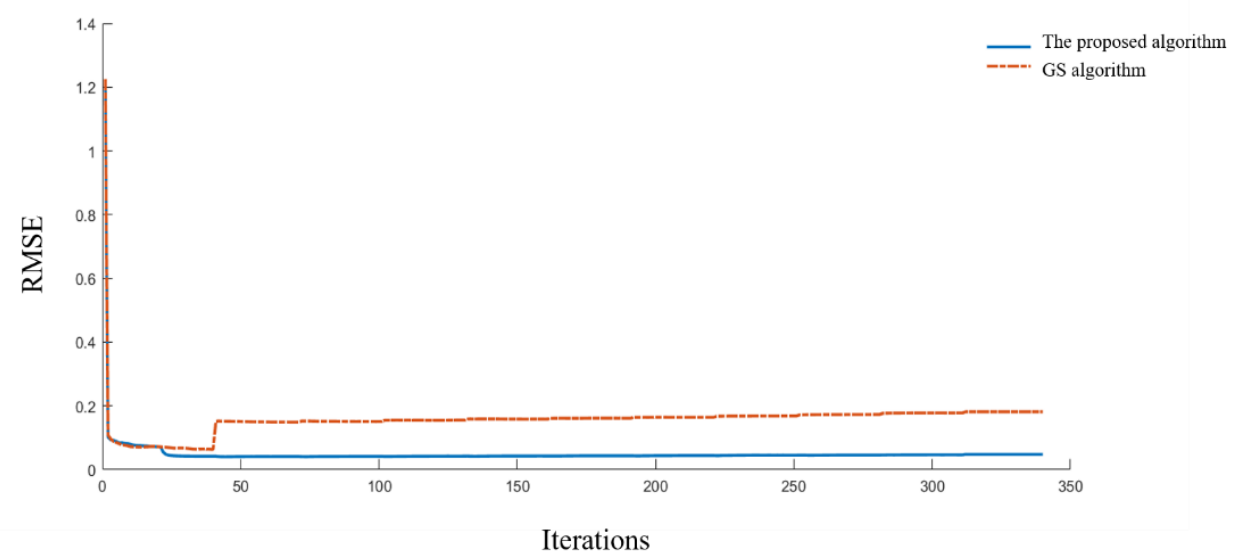

(C) A Comparison of convergence behavior between the proposed algorithm and GS algorithm

Fig.5. Target image and RMSE curves

+20 (the second stage)+500(the third stage)). In Fig.5 (b), the RMSE is small enough when the total number of iteration is 340. To further compare the convergence behavior between GS and the proposed algorithm in this work, the RMSEs of two algorithm against versus the iteration number are plotted in Fig.5(c), respectively. The results suggest that the proposed algorithm can converge to a smaller RMSE than GS algorithm when the soft quantization process is implemented.

The GPU is used to accelerate the calculation speed; the iteration optimization time is about 120 seconds on a computer equipped with Intel Core ${ }^{\mathrm{TM}}$ I7-8750H CPU@2.2GHz, GPU GTX1060 and the computation platform is MATLAB software. The final optimized DOE is shown in Fig.6 (a), the inset is a cross section of the phase value distribution in the red circle. It is clearly indicating an 8 phase-level structure. The simulated reconstructed virtual image is shown in Fig.6 (b), the reconstructed image is similar to the target image in Fig.5 (a). But, the reconstructed image is a bit darker than the target image, because the calculated diffraction efficiency is about $82 \%$, part of the energy being lost as noise. The diffraction efficiency is defined as

$$
\eta=\frac{\sum_{M} \sum_{N} I(I \in S)}{\sum_{M} \sum_{N} I}
$$

Where $I$ is the intensity distribution of the target plane, $M$ and $N$ are the number of sampling points in the $X$ and the $Y$ direction, respectively.

For our application, the aim is to generate horizontal parallax 3D views. 11 off-axis Fresnel type DOE elements are used. The size of the FDOE is $29.7 \mathrm{~mm} \times 2.7 \mathrm{~mm}$, it can be regarded as the system eye-box. However, this is too small to be convenient in the vertical direction, as an observer will no longer see the virtual object if their eye moves beyond this small area. The pupil size of human eye is between $6 \mathrm{~mm} \sim 8 \mathrm{~mm}$, so the vertical direction should be expanded to satisfy this size. To address this problem, we propose a method to increase the vertical eye-box size. The assembly is shown in Fig.7. 


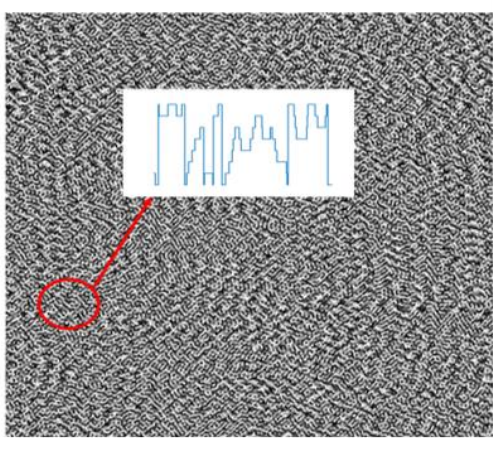

(a) Phase map of the DOE

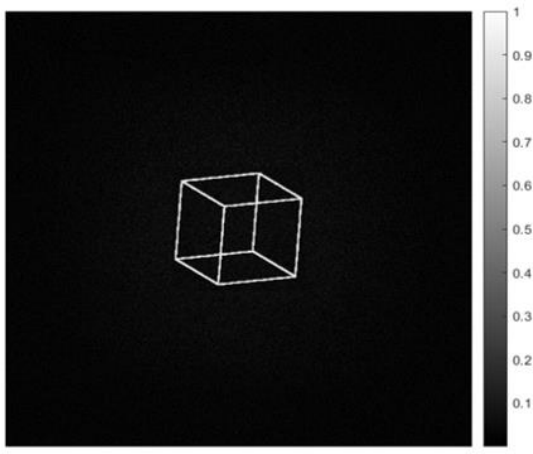

(b) Reconstructed virtual image

Fig.6. Optimal phase map and the reconstructed result

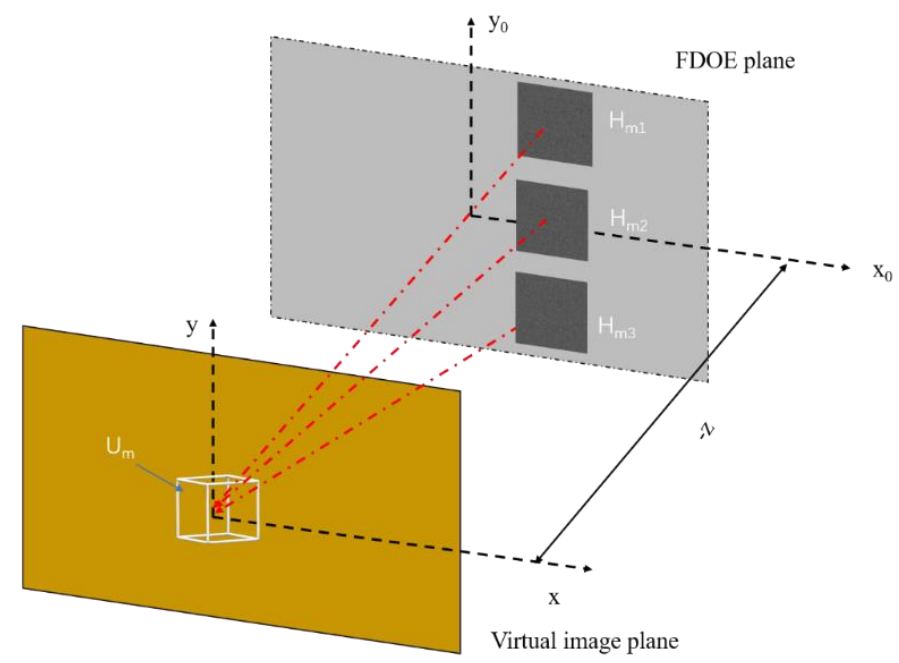

Fig.7. Assemble of the FDOE

In Fig.7, the elements $\mathrm{H}_{\mathrm{m} 0}, \mathrm{H}_{\mathrm{m} 1}$ and $\mathrm{H}_{\mathrm{m} 3}$ generate the same virtual image $\mathrm{U}_{\mathrm{m}}$ at the same position. When people move their eye in vertical direction, they can still see the same virtual image at specific view. In this way, the eyebox can be expanded in vertical direction, and the final size of the FDOE is $29.7 \mathrm{~mm} \times 8.1 \mathrm{~mm}$. Due to the use of divergent illumination and DOE fabrication limitations, the LED source is visible to the observer. If the FDOE are designed with an on axis target, this visibility of the LED source perturbs the desired image. To avoid this, an offaxis configuration is used.

\section{Fabrication and experiment results}

With the algorithm mentioned above, a FDOE is designed with $11 \times 3$ facets. The smallest pixel size of our photolithography machine is $0.75 \mu \mathrm{m}$, but here we use $1.5 \mu \mathrm{m}$ as the design pixel size. It helps minimize pixel deformation in the off-axis configuration which tends to decrease the DOE fringe size. Each DOE facet is $1800 \times 1800$ pixels with pixel size $1.5 \mu \mathrm{m}$, the total FDOE size is $29.7 \mathrm{~mm} \times 8.1 \mathrm{~mm}$. The FDOE was fabricated with our home-built parallel direct-writing photolithography machine, an updated version of that demonstrated in [23-25]. In Fig.8, the light source is LED based with a center wavelength of $435 \mathrm{~nm}$. The divergent light is first homogenized using group of lenses and a diffuser. Then, the collimated and uniform light field is modulated with the designed phase pattern loaded onto a 1920×1080 pixel Spatial Light Modulator(SLM) (Epson L3D135-55g00) and de-magnified onto the surface of the photoresist on the precision moving platform through the objective lens group. The magnification ratio of the objective lens group is 5 . The projected area of SLM is small, about $4 \mathrm{~mm} \times 3.2 \mathrm{~mm}$, so the FDOE is made by 
stitching different SLM images by moving the stage in the $\mathrm{x}$ and $\mathrm{y}$ directions with an accuracy of about $250 \mathrm{~nm}$. The fabrication process is now described briefly. The glass substrate is cleaned with ultrasonic water cleaning, and then heated for 15 minutes to dry the glass on a hot plate. The next step is the spin coating of a photoresist onto the substrate. In this work, the photoresist is the S1813 from Micro Resist Technology GmbH. The spin coating speed is chosen to give uniform photoresist layer. Etch depth is chosen to give the maximum phase difference of each cell is $2 \pi$. The etch depth is dependent on the wavelength $\lambda$ of the incident light and the refractive index of the resist. According to the thin element approximation (TEA), the groove depth of the surface relief structure can be written as

$$
\mathrm{d}=\frac{N-1}{N} \frac{\lambda}{2\left(n_{\text {material }}-n_{\text {air }}\right)}
$$

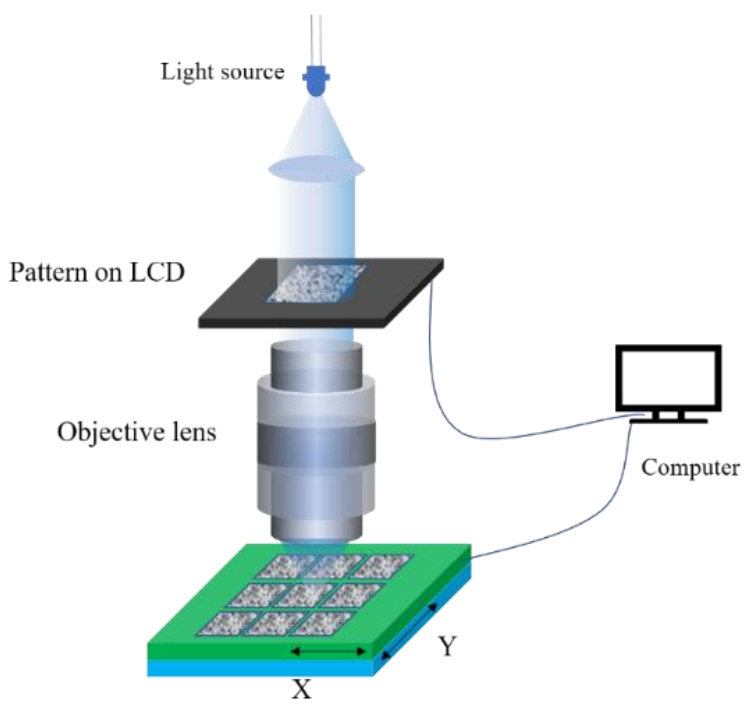

XY Tables

Fig.8. Diagram of photo-lithography system

where number of phase levels, $\mathrm{N}=8, n_{\text {material }}$ is the refractive index of photoresist, its value is 1.66 at a wavelength of $532 \mathrm{~nm}$. According to the formula, the maximum depth $\mathrm{d}$ is about $691 \mathrm{~nm}$. The spin coating speed is $3200 \mathrm{rpm}$, giving a total photoresist thickness about $1.3 \mu \mathrm{m}$; this depth is enough for etching the DOE. The substrate is baked for 2 minutes at temperature of 120 degree to extract the solvent, and then cooled down to room temperature. Then, exposure is implemented using our home-built parallel direct-writing photo-plotter with a lookup table (LUT) to establish a linear relation between the addressed gray phase level and the etch depth. The exposed pattern is mapped from the SLM screen to photoresist plane. This step needs about 15 minutes. The exposure time and LUT can be controlled to ensure the optimum etching depth. The last step is to develop the exposured photoresist in a chemical solution for 2 minutes. A fragment of the FDOE and final fabricated FDOE are shown in Fig.9 (a) and Fig.9 (b), respectively. 


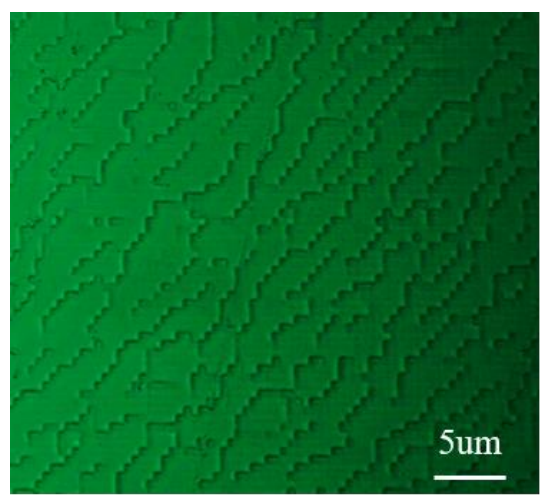

(a)

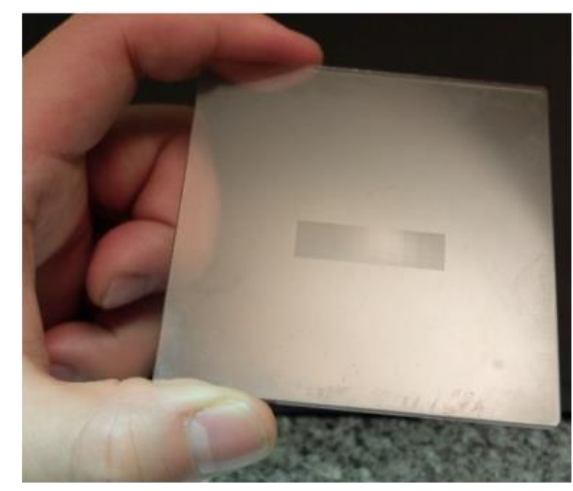

(b)

Fig.9. Fragment part of FDOE and real sample.(a) Fragment of FDOE;(b) Real sample on photoresist with glass substrate.

The photo of Fig.9 (a) is captured by an optical microscope (Reichert-Jung Polyvar Met Microscope), with a 100x objective.

The schematic of experimental set up used to capture the output views is shown in Fig.10. This optical system comprises an LED source, a FDOE and a camera. A green LED source behind the FDOE is used as the illumination source for reproducing the expected 3D virtual image. The camera is mounted on the support, which can be rotated to gain different viewing angles equivalent to those can observer sees moving his head to view the apparent 3D object. The distance between LED and FDOE is about $155 \mathrm{~mm}$, and position of the virtual image is about $70 \mathrm{~mm}$ below the FDOE. The position of virtual image can be calculated according to the imaging formula, which is expressed in eq. (18).

$$
\mathrm{d}_{\text {image }}=\frac{f_{\text {fresnel }} d_{s}}{d_{s}-f_{\text {fresnel }}}
$$

In eq.(18), $f_{\text {fresnel }}$ is the designed focal length of the Fresnel type DOE whose value is $-120 \mathrm{~mm}, d_{s}$ is the distance between the source and the FDOE plane. With the LED light illumination, the light diffracted by the FDOE propagates to human eye and the 3D virtual image is perceived in the air between the DOE plane and the LED source. Fig.11 shows one perspective image of the 3D target "cube". When the hologram is optically replayed, a floating virtual image appears hanging in space behind the FDOE, a finger is near to the virtual image. An observer also can see a realistic 3D image by looking at the virtual object from different angle. Fig.12(a)-(f) shows six reconstructed images of the FDOE, which are captured from six viewing angles by rotating the camera from left to right, see Fig.10. The different views all correspond to a single 3D object located in the same spatial position. The field of view (FOV) is about 20 degree. The brightness of the reconstructed images vary slightly because the exposure time of camera is slightly different for the different image capture angles. In practice, the visual effect perceived by the observer is of a $3 \mathrm{D}$ virtual image with more uniform brightness. 


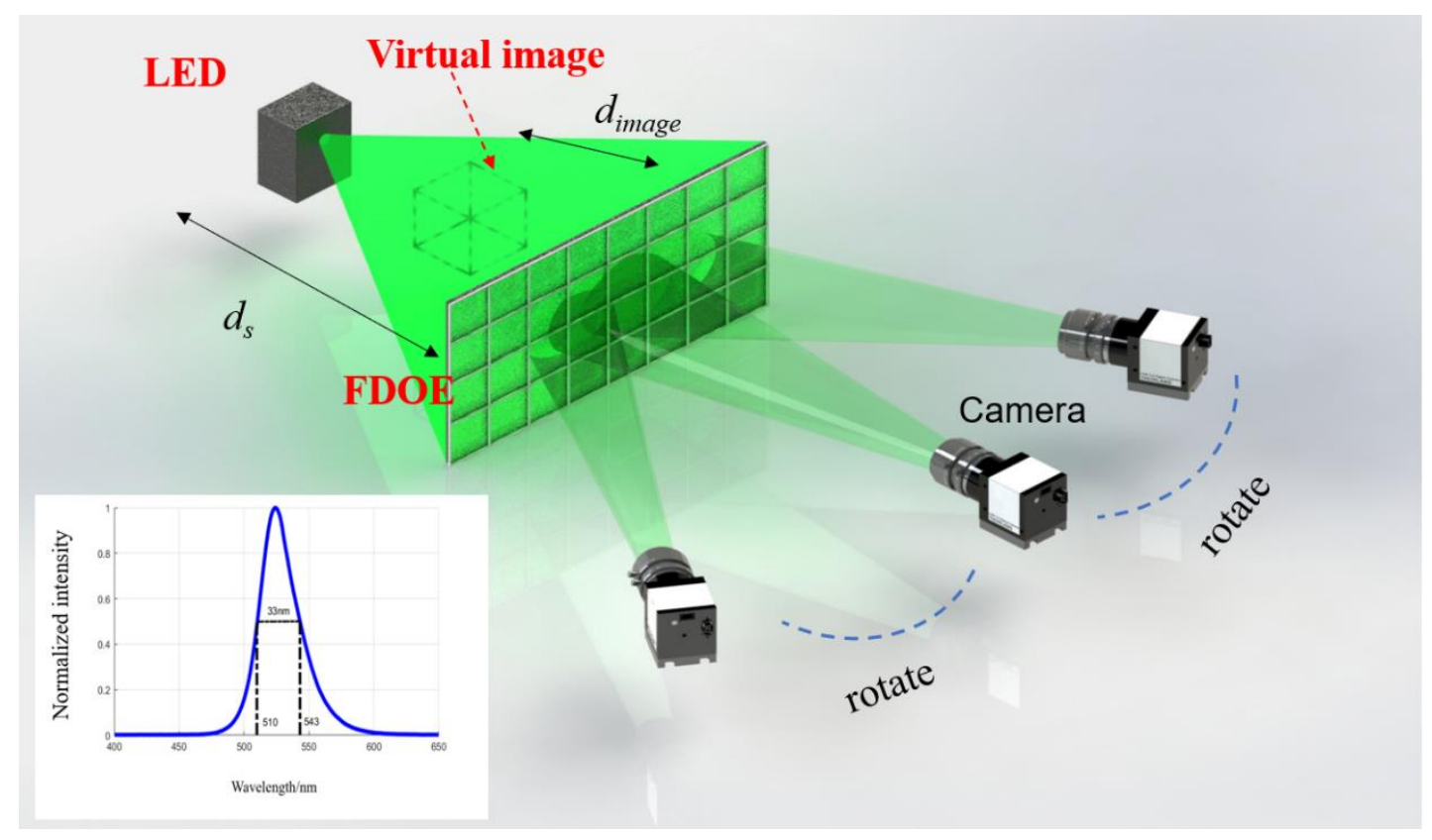

Fig.10. Schematic diagram of capturing the 3D floating virtual object reconstruction. Inset: spectrum of the LED source, which is obtained by Ocean Optics HR4000CG-UV-NIR, its center wavelength is $525 \mathrm{~nm}$.

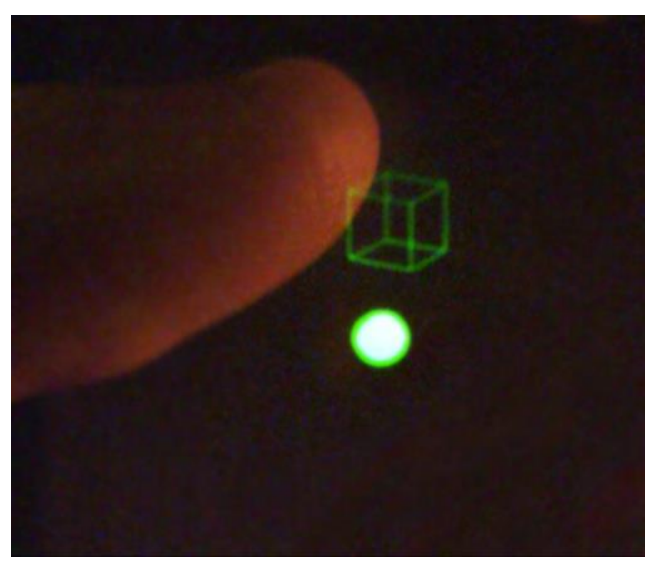

Fig.11. One perspective of the 3D cube floating in the air

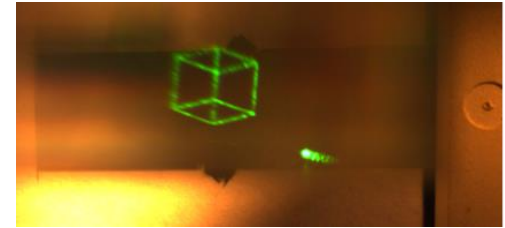

(a)

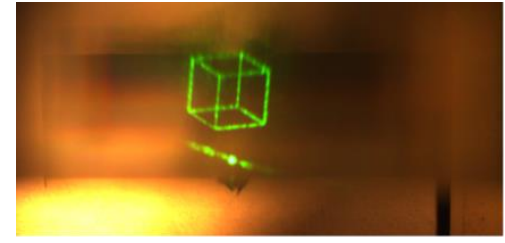

(d)

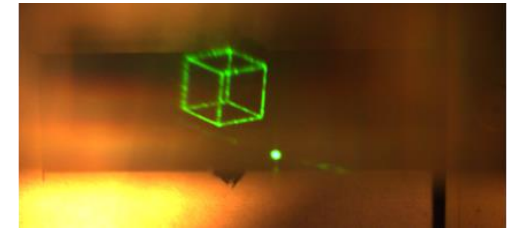

(b)

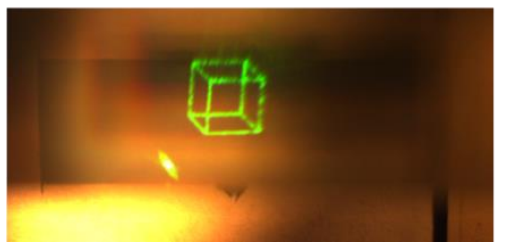

(e)

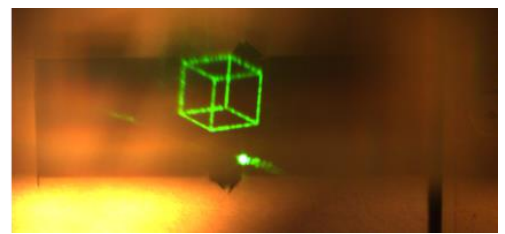

(c)

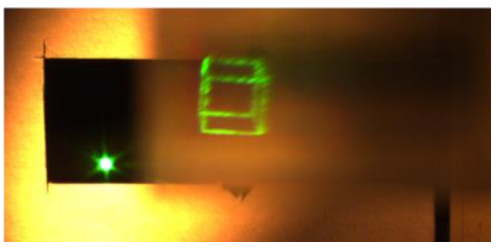

(f)

Fig.12. Different views of the virtual "3D cube" object when the FDOE is observed from different angles with divergent illumination of the FDOE). (See Visualization 1) 


\section{Discussion}

This paper has presented the design process for a diffractive structure, based on an array of computer generated offaxis Fresnel phase holograms. When illuminated by a readily available LED source, the overall structure gives an observer the perception of a floating 3D object in space behind the hologram. The design model has been described and confirmed through both numerical simulation and experimental results. Although, this work focused on presenting the design methodology to generate perceived 3D virtual vision in the horizontal direction, the fullparallax vision can also be generated with the same approach. As illustrated in Fig.7, we just let the $\mathrm{H}_{\mathrm{m} 1}, \mathrm{H}_{\mathrm{m} 2}$ and $\mathrm{H}_{\mathrm{m} 3}$ form the same image at the same position. If we let them produce different views along the vertical direction, the observer can perceives different views in the vertical direction, so the full-parallax vision can be realized in this fashion.

Another important factor is the position of the LED source. In this work, the FDOE we designed is a shiftinvariant component, since the light propagation model used here is a linear transformation; the observed result is not sensitive to the position of LED source. Mathematically, the function of off-axis Fresnel type DOE can be decomposed into three linearly components as a spherical lens, Fourier type DOE and the blazed grating phase. The blazed grating phase component is used to

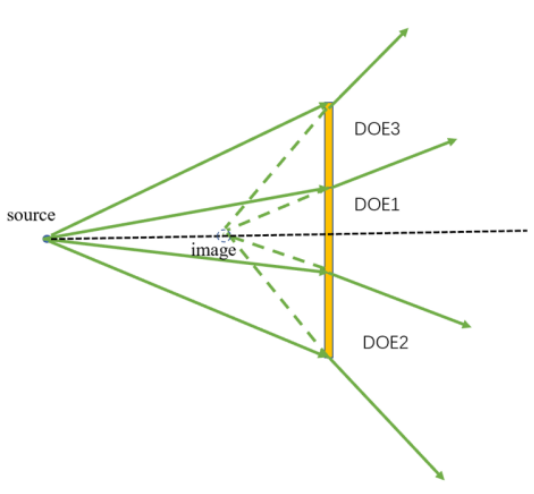

(a)

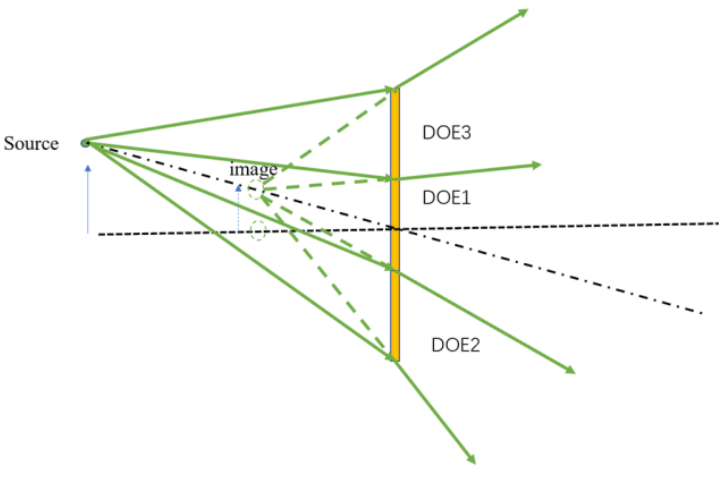

(b)

Fig.13. Schematic of imaging principle, (a) LED source is on the axis, (b) LED source is off axis . shift the image to the desired position. In Fig.13 (a), when the LED source is on the axis of the DOE1, the virtual image is still formed on the axis because there is no blazed grating component in the DOE1. The position of the generated virtual image can be calculated with the geometrical imaging formula of eq. (18). The DOE2 and DOE3 contain the blazed grating component, so, their corresponding virtual image can be deflected to the same position with DOE1. When the LED source is moved with respect to the original position (see Fig.13 (b)), the virtual image will move in the same direction, this process is similar to a lens imaging system, because the Fresnel lens component works as a lens. In this situation, the corresponding images generated by DOE2 and DOE3 just move in the same way.

But, one thing must be borne in mind, the LED source is a divergent source, and the intensity is cosine with the divergent angle. The bigger divergent angle, the weaker the intensity. So, the shifting of the LED source cannot be too far.

Another important factor is the spatial coherence of the LED source, which has been discussed in detail in reference [26]. According to the results, the size of the LED cannot be too big; otherwise, the reconstructed image will be not sharp due to the low degree of spatial coherence. In this work, the LED size is $1 \mathrm{~mm} \times 1 \mathrm{~mm}$, but as the distance from the LED source to FDOE plane is more than $150 \mathrm{~mm}$, the LED source can be still regarded as 'point source'. 
Another essential DOE parameter to be evaluated is the diffraction efficiency: the image must be sufficiently bright to be visible to the observer. Unfortunately measuring the diffraction efficiency of this type of DOE is not straightforward because the image is virtual and the divergent illumination. To obtain an estimate of the diffraction efficiency we used the setup shown in Fig.14. The condenser lens is used to convert the virtual image into a real image which is projected onto the photodetector. A diaphragm is placed between the LED source and the DOE so as to illuminate only the active DOE area. Without the DOE, the total collected light power on the detector gave an estimate of the incident light power. With the DOE present the light power in the desired image area gave an estimate of the light power in the signal. In this way we obtained a DOE diffraction efficiency estimate of $46 \%$. The DOE zeroth order was estimated in a similar way at $20 \%$. The remaining light losses being due mainly to Fresnel reflection $(\sim 10 \%)$ and higher diffraction orders. With this preliminary un-optimized DOE, illuminated with divergent nonmonochromatic (bandwidth $\sim 33 \mathrm{~nm}$ ) light such light losses are not unexpected. We are currently improving our fabrication facilities to decrease the DOE pixel size which will increase diffraction efficiency by suppressing the higher diffraction orders [27] and optimizing the setup and algorithm to make the DOE easier to fabricate. Based on values obtained with similar DOEs, we expect to be able to reduce zero order power to a few percent. However, even with the current diffraction efficiency of $46 \%$, the observed image is already clearly visible in daylight with a readily available LED.

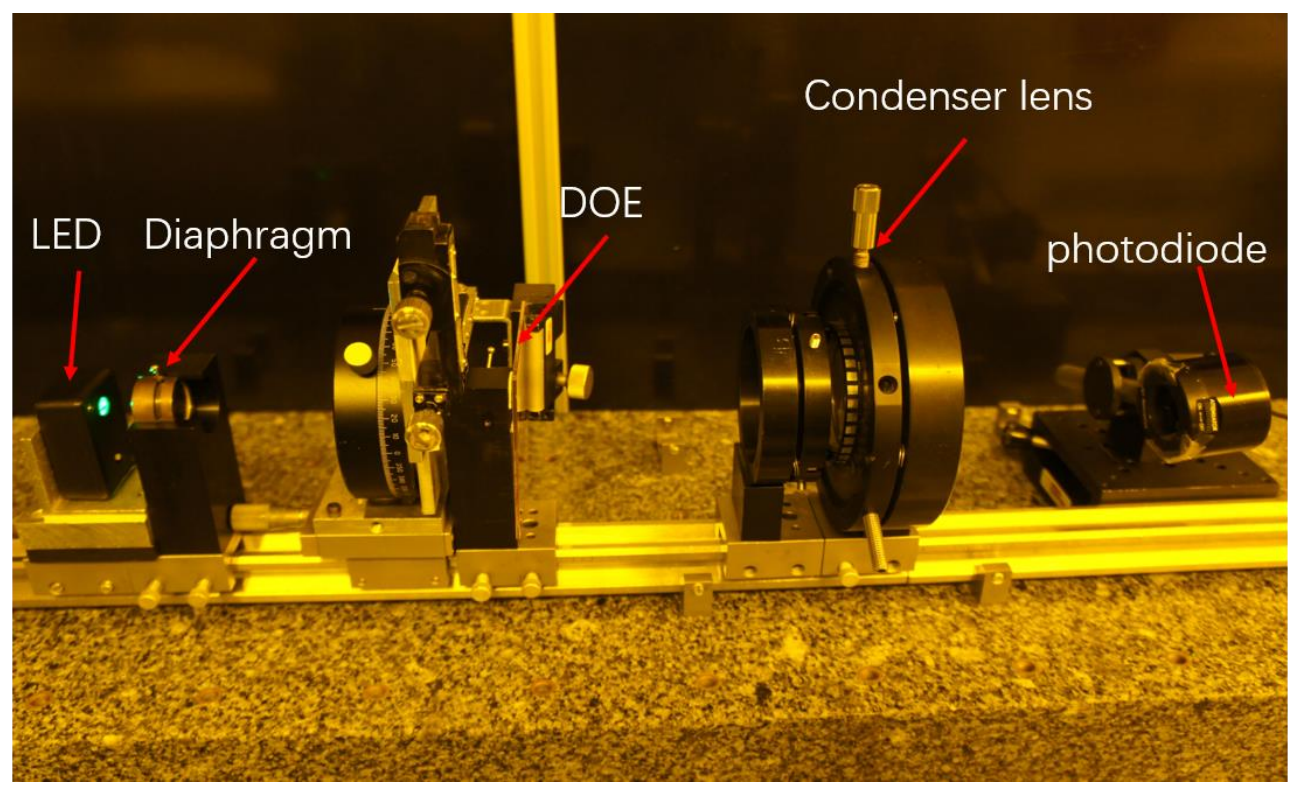

Fig.14. Setup for diffraction parameters measurement

\section{Conclusion}

This paper has presented a computer generated horizontal moving parallax synthetic DOE based on facet Fresnel type DOE array. The design model for the FDOE has been demonstrated through both numerical simulation and experimental results. In this work, we limit viewing of the FDOE to a horizontal displacement of the observer's eye exclusively. A visually convincing 3D virtual object can be observed when the FDOE is illuminated with a divergent LED source. The proposed optimization method can also be implemented to calculate a full-parallax DOE, which has been described in Section 5. As the FDOE is a surface relief diffractive structure, the FDOE can be replicated using roll to roll nanoimprint techniques for mass production. Therefore, the FDOE developed in this paper can find applications in anti-counterfeiting protecting of bank notes and ID cards. The approach may potentially also be used in practical holographic 3D display application. 


\section{Funding}

European Union's Horizon 2020 (780278)

\section{Acknowledgments}

The authors acknowledge the support by the European Union's Horizon 2020 research and innovation program through PHENOmenon under grant agreement No.780278 and Brest Metropole.

\section{References}

1. R. L. Van Renesse, Optical Document Security (Artech House, 2005).

2. Yue, Z. J., Xue, G. L., Liu, J., Wang, Y. T. \& Gu, M. Nanometric holograms based on a topological insulator material. Nature Commu 8, 5(2017).

3. L. C. Ferri, "Visualization of 3D information with digital holography using laser printers," Comput. Graph.25(2), 309-321 (2001).

4. C. K. Lee, J. W. Wu, S. L. Yeh, C. W. Tu, Y. A. Han, E. H. Liao, L. Y. Chang, I. E. Tsai, H. H. Lin, J. C. Hsieh, and J. T. Lee, "Optical configuration and color-representation range of a variable-pitch dot matrix holographic printer," Appl. Opt. 39(1), 40-53 (2000)

5. Kevin T.P. Lim, Hailong Li, Yejing Liu and Joel K.W. Yang, "Holographic colour prints for enhanced optical security by combined phase and amplitude control," Nat. Commu 10, 25(2019).

6. Mindaugas Andrulevicius. et al. , "Hologram Origination Combining Rainbow and Dot-matrix Holograms," Materials Science 16(4), 298-301 (2010).

7. Vasconcellos, F. D. et al. , "Printable Surface Holograms via Laser Ablation," ACS Photonics 1, 489-495(2014).

8. An. Firsov, A. Firsov, B. Loechel, A. Erko, A. Svintsov, and S. Zaitsev, “ Fabrication of digital rainbow holograms and 3-D imaging using SEM based e-beam lithography,” Opt. Express. 22, 28756-28770(2014).

9. M. Skeren, P. Fiala, and I. Richter, "Synthetic diffractive elements for security applications realized on an enhanced integral dot-matrix system,” Appl. Opt. 45(1), 27-32 (2006).

10. R. A. Lee, "Pixelgram: an application of electron-beam lithography for the security printing industry," Proc. SPIE 1509, 48-54 (1991)

11. M. J. Huang, S. L. Yeh, C. K. Lee, and T. K. Huang, "Large-format grating image hologram based on e-beam lithography," Proc. SPIE 2652, 117-123 (1996).

12. V. I. Girnyk, V. I. Grygoruk, I. S. Borisov, S. A. Kostyukevych, and V. Lashkaryov, "Stereographic and animated rainbow diffractive images in optical security,” Proc. SPIE 5290, 179-189 (2004)

13. Alexander Goncharsky, Anton Goncharsky, and Svyatoslav Durlevich, "Diffractive optical element with asymmetric microrelief for creating visual security features,” Opt. Express 23, 29184-29192(2015).

14. Alexander Goncharsky, Anton Goncharsky, and Svyatoslav Durlevich, "Diffractive optical element for creating visual 3D images,” Opt. Express. 24, 9140-9148(2016).

15. Alexander Goncharsky, Anton Goncharsky, and Svyatoslav Durlevich, " High-resolution full-parallax computergenerated holographic stereogram created by e-beam technology," Opt. Eng. 56(6),063105(2017).

16. Anton Goncharsky and Svyatoslav Durlevich, "Cylindrical computer-generated hologram for displaying 3D images," Opt. Express. 26, 22160-22167(2018).

17. Xin Yang, Hui Wang, Yong Li, FuYang Xu, HongBo Zhang, JiaHeng Zhang, QianHong Yan, “ Computer generated full-parallax synthetic hologram based on frequency mosaic," Opt. Commun. 430, 24-30(2019).

18. G.N.Nguyen, K.Heggarty, P. Gerad, B. Serio, and P. Meyrueis, "Computational efficient scalar non-paraxial modeling of optical wave propagation in the far-field,” Appl. Opt. 53, 2196-2205(2014).

19. Huang Hao, Zhai Tingting, Song Qiang, Yin Xiaodong, “ Wide angle 2D bean splitter design based on vector 
diffraction theory," Opt. Commun. 434, 28-35(2019).

20. Haichao Wang, Weirui, Yue, Qiang Song, Jingdan Liu, Guohai Situ, “ A hybrid Gerchberg-Saxton-like algorithm for DOE and CGH calculation,” Opt. Lasers Eng. 89,109-115(2017).

21. Frank Wyrowski, "Diffractive optical elements: iterative calculation of quantized, blazed phase structures," J. Opt. Soc. Am. A 7,961(1990).

22. Giang-Nam Nguyen, Kevin Heggarty, Andreas Bacher, Peter-Jürgen Jakobs, Daniel Häringer, Philippe Gérard, Pierre Pfeiffer, and Patrick Meyrueis, "Iterative scalar nonparaxial algorithm for the design of Fourier phase elements," Opt. Letter. 39, 5551-5554(2014).

23. Melanie V. Kessels, Marwa Elbouz ,Robin Pagan, Kevin Heggarty, “ Versatile stepper based maskless microlithography using a liquid crystal display for direct write of binary and multilevel microstructures," J. of Micro/Nanolithography, MEMS, and MOEMS, 6(3), 033002 (2007).

24. M.V.Kessels, Kevin Heggarty, "Optical proximity correction for a versatile LCD based direct write maskless photoplotter," Microelectronic Engineering. 86, 2385-2391(2009).

25. M.V.Kessels, C.Nassour, P.Grosso, K.Heggarty, "Direct write of optical diffractive elements and planar waveguides with a digital micromirror device based UV photoplotter," Opt. Commun. 283, 3089-3094(2010).

26. Yuanbo Deng, Daping Chu, "Coherence properties of different light sources and their effect on the image sharpness and speckle of holographic displays,” Sci. Rep. 7,5893(2017).

27. H. Schwarzer, S.Teiwes, Frank Wyrowski, “ Non-pixelated” design of computer-generated diffractive elements for increased diffraction efficiency," EOS Topical meeting on Diffractive Optics. 164-165(1997). 